\title{
Common fixed points of Ćirić-type contractive mappings in two ordered generalized metric spaces
}

\author{
M Abbas ${ }^{1}$, YJ Cho ${ }^{2}$ and T Nazir ${ }^{*}$
}

\section{"Correspondence:}

talat@lums.edu.pk

'Department of Mathematics, Lahore University of Management Sciences, Lahore, 54792, Pakistan ${ }^{2}$ Department of Mathematics Education and the RINS, Gyeongsang National University, Jinju, 660-701, Korea

\begin{abstract}
In this paper, using the setting of two ordered generalized metric spaces, a unique common fixed point of four mappings satisfying a generalized contractive condition is obtained. We also present an example to demonstrate the results presented herein. MSC: $54 \mathrm{H} 25 ; 47 \mathrm{H} 10 ; 54 \mathrm{E} 50$
\end{abstract}

Keywords: weakly compatible mappings; compatible mappings; dominated mappings; common fixed point; partially ordered set; generalized metric space

\section{Introduction and preliminaries}

The study of a unique common fixed point of given mappings satisfying certain contractive conditions has been at the center of rigorous research activity. Mustafa and Sims [1] generalized the concept of a metric in which a real number is assigned to every triplet of an arbitrary set. Based on the notion of generalized metric spaces, Mustafa et al. [2-5] obtained some fixed point theorems for some mappings satisfying different contractive conditions. The existence of common fixed points in generalized metric spaces was initiated by Abbas and Rhoades [6] (see also [7] and [8]). For further study of common fixed points in generalized metric spaces, we refer to [9-12] and references mentioned therein. Abbas et al. [13] showed the existence of coupled common fixed points in two generalized metric spaces (for more results on couple fixed points, see also [14-21]).

The existence of fixed points in ordered metric spaces has been initiated in 2004 by Ran and Reurings [22] and further studied by Nieto and Lopez [23]. Subsequently, several interesting and valuable results have appeared in this direction [24-30].

The aim of this paper is to study common fixed point of four mappings that satisfy the generalized contractive condition in two ordered generalized metric spaces.

In the sequel, $\mathbb{R}, \mathbb{R}^{+}$and $\mathbb{N}$ denote the set of real numbers, the set of nonnegative integers and the set of positive integers respectively. The usual order on $\mathbb{R}$ (respectively, on $\mathbb{R}^{+}$) will be indistinctly denoted by $\leq$ or by $\geq$.

In [1], Mustafa and Sims introduced the following definitions and results:

Definition 1.1 Let $X$ be a nonempty set. Suppose that a mapping $G: X \times X \times X \rightarrow \mathbb{R}^{+}$ satisfies the following conditions:

(a) $G(x, y, z)=0$ if $x=y=z$ for all $x, y, z \in X$;

(b) $0<G(x, y, z)$ for all $x, y, z \in X$ with $x \neq y$;

o 2012 Abbas et al.: licensee Springer. This is an Open Access article distributed under the terms of the Creative Commons Attribution License (http://creativecommons.org/licenses/by/2.0), which permits unrestricted use, distribution, and reproduction in any medium, provided the original work is properly cited. 
(c) $G(x, x, y) \leq G(x, y, z)$ for all $x, y, z \in X$ with $y \neq z$;

(d) $G(x, y, z)=G(p\{x, y, z\})$, where $p$ is a permutation of $x, y, z \in X$ (symmetry);

(e) $G(x, y, z) \leq G(x, a, a)+G(a, y, z)$ for all $x, y, z, a \in X$.

Then $G$ is called a $G$-metric on $X$ and $(X, G)$ is called a $G$-metric space.

Definition 1.2 A sequence $\left\{x_{n}\right\}$ in a $G$-metric space $X$ is called:

(1) a G-Cauchy sequence if, for any $\varepsilon>0$, there exists $n_{0} \in N$ (the set of natural numbers) such that, for all $n, m, l \geq n_{0}, G\left(x_{n}, x_{m}, x_{l}\right)<\varepsilon$;

(2) G-convergent if, for any $\varepsilon>0$, there exist $x \in X$ and $n_{0} \in N$ such that, for all $n, m \geq n_{0}, G\left(x, x_{n}, x_{m}\right)<\varepsilon$

(3) A G-metric space $X$ is said to be G-complete if every G-Cauchy sequence in $X$ is G-convergent in $X$.

It is known that $\left\{x_{n}\right\}$ is $G$-convergent to a point $x \in X$ if and only if $G\left(x_{m}, x_{n}, x\right) \rightarrow 0$ as $n, m \rightarrow \infty$.

Proposition 1.3 [1] Let $X$ be a G-metric space. Then the following items are equivalent:

(1) A sequence $\left\{x_{n}\right\}$ in $X$ is G-convergent to a point $x \in X$;

(2) $G\left(x_{n}, x_{m}, x\right) \rightarrow 0$ as $n, m \rightarrow \infty$;

(3) $G\left(x_{n}, x_{n}, x\right) \rightarrow 0$ as $n \rightarrow \infty$;

(4) $G\left(x_{n}, x, x\right) \rightarrow 0$ as $n \rightarrow \infty$.

Definition 1.4 A $G$-metric on $X$ is said to be symmetric if $G(x, y, y)=G(y, x, x)$ for all $x, y \in X$.

Proposition 1.5 Every G-metric on $X$ defines a metric $d_{G}$ on $X$ by

$$
d_{G}(x, y)=G(x, y, y)+G(y, x, x)
$$

for all $x, y \in X$.

For a symmetric G-metric, we have

$$
d_{G}(x, y)=2 G(x, y, y)
$$

for all $x, y \in X$. However, if $G$ is non-symmetric, then the following inequality holds:

$$
\frac{3}{2} G(x, y, y) \leq d_{G}(x, y) \leq 3 G(x, y, y)
$$

for all $x, y \in X$. It is obvious that

$$
G(x, x, y) \leq 2 G(x, y, y)
$$

for all $x, y \in X$.

Now, we give an example of a non-symmetric $G$-metric. 
Table 1 G-metric

\begin{tabular}{ll}
\hline $\mathbf{( x , y , z )}$ & $\mathbf{G ( x , y , z )}$ \\
\hline$(1,1,1),(2,2,2)$ & 0 \\
$(1,1,2),(1,2,1),(2,1,1)$ & 0.5 \\
$(1,2,2),(2,1,2),(2,2,1)$ & 1.0 \\
\hline
\end{tabular}

Example 1.6 Let $X=\{1,2\}$ and $G: X \times X \times X \rightarrow \mathbb{R}^{+}$be a mapping defined by Table 1 .

Note that $G$ satisfies all the axioms of a generalized metric, but $G(x, x, y) \neq G(x, y, y)$ for two distinct points $x, y \in X$.

Definition 1.7 Let $f$ and $g$ be self-mappings on a set $X$. If $w=f x=g x$ for some $x \in X$, then the point $x$ is called a coincidence point of $f$ and $g$ and $w$ is called a point of coincidence of $f$ and $g$.

Definition 1.8 [31] Let $f$ and $g$ be self-mappings on a set $X$. Then $f$ and $g$ are said to be weakly compatible if they commute at every coincidence point.

Definition 1.9 [8] Let $X$ be a $G$-metric space and $f, g$ be self-mappings on $X$. Then $f$ and $g$ are said to be $R$-weakly commuting if there exists a positive real number $R$ such that $G(f g x, f g x, g f x) \leq R G(f x, f x, g x)$ for all $x \in X$.

The maps $f$ and $g$ are $R$-weakly commuting on $X$ if and only if they commute at their coincidence points.

Recall that two mappings $f$ and $g$ on a $G$-metric space $X$ are said to be compatible if, for a sequence $\left\{x_{n}\right\}$ in $X$ such that $\left\{x_{n}\right\}$ and $\left\{g x_{n}\right\}$ are $G$-convergent to some $t \in X$,

$$
\lim _{n \rightarrow \infty} G\left(f g x_{n}, f g x_{n}, g f x_{n}\right)=0 .
$$

Definition 1.10 Let $X$ be a nonempty set. Then $(X, \preceq, G)$ is called an ordered generalized metric space if the following conditions hold:

(a) $G$ is a generalized metric on $X$;

(b) $\preceq$ is a partial order on $X$.

Definition 1.11 Let $(X, \preceq)$ be a partial ordered set. Then two points $x, y \in X$ are said to be comparable if $x \leq y$ or $y \leq x$.

Definition 1.12 [24] Let $(X, \preceq)$ be a partially ordered set. A self-mapping $f$ on $X$ is said to be dominating if $x \preceq f x$ for all $x \in X$.

Example 1.13 [24] Let $X=[0,1]$ be endowed with usual ordering and $f: X \rightarrow X$ be a mapping defined by $f x=\sqrt[n]{x}$ for some $n \in \mathbb{N}$. Since $x \leq x^{\frac{1}{n}}=f x$ for all $x \in X, f$ is a dominating mapping.

Definition 1.14 Let $(X, \preceq)$ be a partially ordered set. A self-mapping $f$ on $X$ is said to be dominated if $f x \preceq x$ for all $x \in X$.

Example 1.15 Let $X=[0,1]$ be endowed with usual ordering and $f: X \rightarrow X$ be a mapping defined by $f x=x^{n}$ for some $n \in \mathbb{N}$. Since $f x=x^{n} \leq x$ for all $x \in X, f$ is a dominated mapping. 
Definition 1.16 A subset $\mathcal{K}$ of a partially ordered set $X$ is said to be well-ordered if every two elements of $\mathcal{K}$ are comparable.

\section{Common fixed point theorems}

In [32], Kannan proved a fixed point theorem for a single valued self-mapping $T$ on a metric space $X$ satisfying the following property:

$$
d(T x, T y) \leq h\{d(x, T x)+d(y, T y)\}
$$

for all $x, y \in X$, where $h \in\left[0, \frac{1}{2}\right)$. If a self-mapping $T$ on a metric space $X$ satisfies the following property:

$$
d(T x, T y) \leq a d(x, y)+b d(x, T x)+c d(y, T y)+e[d(x, T y)+d(y, T x)]
$$

for all $x, y \in X$, where $a, b, c, e \geq 0$ with $a+b+c+2 e<1$, then $T$ has a unique fixed point provided that $X$ is $T$-orbitally complete (for related definitions and results, we refer to [33]).

Afterwards, Ćirić [34] obtained a fixed point result for a mapping satisfying the following property:

$$
d(T x, T y) \leq q \max \left\{d(x, y), d(x, T x), d(y, T y), \frac{d(x, T y)+d(y, T x)}{2}\right\}
$$

for all $x, y \in X$, where $0 \leq q<1$.

In this section, we show the existence of a unique common fixed point of four mappings satisfying Cirić-type contractive condition in the framework of two ordered generalized metric spaces.

Now, we start with the following result:

Theorem 2.1 Let $(X, \preceq)$ be a partially ordered set and $G_{1}, G_{2}$ be two G-metrics on $X$ such that $G_{2}(x, y, z) \leq G_{1}(x, y, z)$ for all $x, y, z \in X$ with a complete metric $G_{1}$ on $X$. Suppose that $f, g, S$ and $T$ are self-mappings on $X$ satisfying the following properties:

$$
\begin{aligned}
G_{1}(f x, f x, g y) \leq & k \max \left\{G_{2}(S x, S x, T y), G_{2}(f x, f x, S x), G_{2}(g y, g y, T y),\right. \\
& {\left.\left[G_{2}(f x, f x, T y)+G_{2}(g y, g y, S x)\right] / 2\right\} }
\end{aligned}
$$

and

$$
\begin{aligned}
G_{1}(f x, g y, g y) \leq & k \max \left\{G_{2}(S x, T y, T y), G_{2}(f x, S x, S x), G_{2}(g y, T y, T y),\right. \\
& {\left.\left[G_{2}(f x, T y, T y)+G_{2}(g y, S x, S x)\right] / 2\right\} }
\end{aligned}
$$

for all comparable $x, y \in X$, where $k \in[0,1)$. Suppose that $f(X) \subseteq T(X)$ and $g(X) \subseteq S(X)$, $f, g$ are dominated mappings and $S, T$ are dominating mappings. If, for any nonincreasing sequence $\left\{x_{n}\right\}$ in $X$ with $y_{n} \preceq x_{n}$ for all $n \in \mathbb{N}, y_{n} \rightarrow u$ implies that $u \preceq x_{n}$ and either

(a) $f$, $S$ are compatible, $f$ or $S$ is continuous and $g, T$ are weakly compatible 
(b) $g, T$ are compatible, $g$ or $T$ is continuous and $f, S$ are weakly compatible,

then $f, g, S$ and $T$ have a common fixed point. Moreover, the set of common fixed points of $f, g, S$ and $T$ is well-ordered if and only iff, $g, S$ and $T$ have one and only one common fixed point.

Proof Let $x_{0}$ be an arbitrary point in $X$. Since $f(X) \subseteq T(X)$ and $g(X) \subseteq S(X)$, we can define the sequences $\left\{x_{n}\right\}$ and $\left\{y_{n}\right\}$ in $X$ by

$$
y_{2 n}=g x_{2 n}=S x_{2 n+1}, \quad y_{2 n+1}=f x_{2 n+1}=T x_{2 n+2}
$$

for all $n \geq 0$. By the given assumptions, we have

$$
\begin{aligned}
& x_{2 n+2} \preceq T x_{2 n+2}=f x_{2 n+1} \preceq x_{2 n+1}, \\
& x_{2 n+1} \preceq S x_{2 n+1}=g x_{2 n} \preceq x_{2 n} .
\end{aligned}
$$

Thus, for all $n \geq 0$, we have $x_{n+1} \preceq x_{n}$. Suppose that $G_{1}\left(y_{2 n}, y_{2 n+1}, y_{2 n+1}\right)>0$ for all $n \geq 0$. If not, then, for some $m \geq 0, y_{m}=y_{m+1}$. Indeed, if $m=2 k$, then $y_{2 k}=y_{2 k+1}$ and from (2.1), it follows that

$$
\begin{aligned}
& G_{1}\left(y_{2 k+1}, y_{2 k+1}, y_{2 k+2}\right) \\
&=G_{1}\left(f x_{2 k+1}, f x_{2 k+1}, g x_{2 k+2}\right) \\
& \leq k \max \left\{G_{2}\left(S x_{2 k+1}, S x_{2 k+1}, T x_{2 k+2}\right), G_{2}\left(f x_{2 k+1}, f x_{2 k+1}, S x_{2 k+1}\right),\right. \\
& G_{2}\left(g x_{2 k+2}, g x_{2 k+2}, T x_{2 k+2}\right), \\
& {\left.\left[G_{2}\left(f x_{2 k+1}, f x_{2 k+1}, T x_{2 k+2}\right)+G_{2}\left(g x_{2 k+2}, g x_{2 k+2}, S x_{2 k+1}\right)\right] / 2\right\} } \\
&= k \max \left\{G_{2}\left(y_{2 k}, y_{2 k}, y_{2 k+1}\right), G_{2}\left(y_{2 k+1}, y_{2 k+1}, y_{2 k}\right), G_{2}\left(y_{2 k+2}, y_{2 k+2}, y_{2 k+1}\right),\right. \\
& {\left.\left[G_{2}\left(y_{2 k+1}, y_{2 k+1}, y_{2 k+1}\right)+G_{2}\left(y_{2 k+2}, y_{2 k+2}, y_{2 k}\right)\right] / 2\right\} } \\
& \leq k \max \left\{G_{2}\left(y_{2 k}, y_{2 k}, y_{2 k+1}\right), G_{2}\left(y_{2 k+1}, y_{2 k+1}, y_{2 k}\right), G_{2}\left(y_{2 k+2}, y_{2 k+2}, y_{2 k+1}\right),\right. \\
& {\left.\left[G_{2}\left(y_{2 k+2}, y_{2 k+2}, y_{2 k+1}\right)+G_{2}\left(y_{2 k+1}, y_{2 k+1}, y_{2 k}\right)\right] / 2\right\} } \\
& \leq k \max \left\{G_{1}\left(y_{2 k}, y_{2 k}, y_{2 k+1}\right), G_{1}\left(y_{2 k+1}, y_{2 k+1}, y_{2 k}\right), G_{1}\left(y_{2 k+2}, y_{2 k+2}, y_{2 k+1}\right),\right. \\
& {\left.\left[G_{1}\left(y_{2 k+2}, y_{2 k+2}, y_{2 k+1}\right)+G_{1}\left(y_{2 k+1}, y_{2 k+1}, y_{2 k}\right)\right] / 2\right\} } \\
&= k G_{1}\left(y_{2 k+2}, y_{2 k+2}, y_{2 k+1}\right) .
\end{aligned}
$$

Again, from (2.2), it follows that

$$
\begin{aligned}
& G_{1}\left(y_{2 k+1}, y_{2 k+2}, y_{2 k+2}\right) \\
&= G_{1}\left(f x_{2 k+1}, g x_{2 k+2}, g x_{2 k+2}\right) \\
& \leq k \max \left\{G_{2}\left(S x_{2 k+1}, T x_{2 k+2}, T x_{2 k+2}\right), G_{2}\left(f x_{2 k+1}, S x_{2 k+1}, S x_{2 k+1}\right),\right. \\
& G_{2}\left(g x_{2 k+2}, T x_{2 k+2}, T x_{2 k+2}\right), \\
& {\left.\left[G_{2}\left(f x_{2 k+1}, T x_{2 k+2}, T x_{2 k+2}\right)+G_{2}\left(g x_{2 k+2}, S x_{2 k+1}, S x_{2 k+1}\right)\right] / 2\right\} }
\end{aligned}
$$




$$
\begin{aligned}
= & k \max \left\{G_{2}\left(y_{2 k}, y_{2 k+1}, y_{2 k+1}\right), G_{2}\left(y_{2 k+1}, y_{2 k}, y_{2 k}\right), G_{2}\left(y_{2 k+2}, y_{2 k+1}, y_{2 k+1}\right),\right. \\
& {\left.\left[G_{2}\left(y_{2 k+1}, y_{2 k+1}, y_{2 k+1}\right)+G_{2}\left(y_{2 k+2}, y_{2 k}, y_{2 k}\right)\right] / 2\right\} } \\
\leq & k \max \left\{G_{2}\left(y_{2 k}, y_{2 k+1}, y_{2 k+1}\right), G_{2}\left(y_{2 k+1}, y_{2 k}, y_{2 k}\right), G_{2}\left(y_{2 k+2}, y_{2 k+1}, y_{2 k+1}\right),\right. \\
& {\left.\left[G_{2}\left(y_{2 k+2}, y_{2 k+1}, y_{2 k+1}\right)+G_{2}\left(y_{2 k+1}, y_{2 k}, y_{2 k}\right)\right] / 2\right\} } \\
\leq & k \max \left\{G_{1}\left(y_{2 k}, y_{2 k}, y_{2 k+1}\right), G_{2}\left(y_{2 k+1}, y_{2 k}, y_{2 k}\right), G_{1}\left(y_{2 k+2}, y_{2 k+1}, y_{2 k+1}\right),\right. \\
& {\left.\left[G_{1}\left(y_{2 k+2}, y_{2 k+1}, y_{2 k+1}\right)+G_{1}\left(y_{2 k+1}, y_{2 k}, y_{2 k}\right)\right] / 2\right\} } \\
= & k G_{1}\left(y_{2 k+2}, y_{2 k+1}, y_{2 k+1}\right) .
\end{aligned}
$$

Thus (2.3) and (2.4) imply that

$$
G_{1}\left(y_{2 k+2}, y_{2 k+1}, y_{2 k+1}\right) \leq k^{2} G_{1}\left(y_{2 k+2}, y_{2 k+1}, y_{2 k+1}\right)
$$

and so $y_{2 k+1}=y_{2 k+2}$ since $k^{2}<1$.

Similarly, if $m=2 k+1$, then one can easily obtain $y_{2 k+2}=y_{2 k+3}$. Thus $\left\{y_{n}\right\}$ becomes a constant sequence and $y_{2 n}$ serves as the common fixed point of $f, g, S$ and $T$.

Suppose that $G_{1}\left(y_{2 n}, y_{2 n+1}, y_{2 n+1}\right)>0$ for all $n \geq 0$.

If $n \in \mathbb{N}$ is even, then $n=2 k$ for some $k \in \mathbb{N}$; then it follows from (2.1) that

$$
\begin{aligned}
& G_{1}\left(y_{n+1}, y_{n+1}, y_{n}\right) \\
&=G_{1}\left(y_{2 k+1}, y_{2 k+1}, y_{2 k}\right) \\
&=G_{1}\left(f x_{2 k+1}, f x_{2 k+1}, g x_{2 k}\right) \\
& \leq k \max \left\{G_{2}\left(S x_{2 k+1}, S x_{2 k+1}, T x_{2 k}\right), G_{2}\left(f x_{2 k+1}, f x_{2 k+1}, S x_{2 k+1}\right),\right. \\
&\left.G_{2}\left(g x_{2 k}, g x_{2 k}, T x_{2 k}\right),\left[G_{2}\left(f x_{2 k+1}, f x_{2 k+1}, T x_{2 k}\right)+G_{2}\left(g x_{2 k}, g x_{2 k}, S x_{2 k+1}\right)\right] / 2\right\} \\
&= k \max \left\{G_{2}\left(y_{2 k}, y_{2 k}, y_{2 k-1}\right), G_{2}\left(y_{2 k+1}, y_{2 k+1}, y_{2 k}\right),\right. \\
&\left.G_{2}\left(y_{2 k}, y_{2 k}, y_{2 k-1}\right),\left[G_{2}\left(y_{2 k+1}, y_{2 k+1}, y_{2 k-1}\right)+G_{2}\left(y_{2 k}, y_{2 k}, y_{2 k}\right)\right] / 2\right\} \\
& \leq k \max \left\{G_{2}\left(y_{2 k}, y_{2 k}, y_{2 k-1}\right), G_{2}\left(y_{2 k+1}, y_{2 k+1}, y_{2 k}\right),\right. \\
& {\left.\left[G_{2}\left(y_{2 k+1}, y_{2 k+1}, y_{2 k}\right)+G_{2}\left(y_{2 k}, y_{2 k}, y_{2 k-1}\right)\right] / 2\right\} } \\
& \leq k \max \left\{G_{1}\left(y_{n}, y_{n}, y_{n-1}\right), G_{1}\left(y_{n+1}, y_{n+1}, y_{n}\right)\right\},
\end{aligned}
$$

which implies that

$$
G_{1}\left(y_{n+1}, y_{n+1}, y_{n}\right) \leq k G_{1}\left(y_{n}, y_{n}, y_{n-1}\right)
$$

If $n \in \mathbb{N}$ is odd, then $n=2 k+1$ for some $k \in \mathbb{N}$. Again, it follows from (2.1) that

$$
\begin{aligned}
& G_{1}\left(y_{n+1}, y_{n+1}, y_{n}\right) \\
& \quad=G_{1}\left(y_{2 k+2}, y_{2 k+2}, y_{2 k+1}\right) \\
& \quad=G_{1}\left(f x_{2 k+2}, f x_{2 k+2}, g x_{2 k+1}\right) \\
& \quad \leq k \max \left\{G_{2}\left(S x_{2 k+2}, S x_{2 k+2}, T x_{2 k+1}\right), G_{2}\left(f x_{2 k+2}, f x_{2 k+2}, S x_{2 k+2}\right),\right.
\end{aligned}
$$




$$
\begin{aligned}
& G_{2}\left(g x_{2 k+1}, g x_{2 k+1}, T x_{2 k+1}\right), \\
& {\left.\left[G_{2}\left(f x_{2 k+2}, f x_{2 k+2}, T x_{2 k+1}\right)+G_{2}\left(g x_{2 k+1}, g x_{2 k+1}, S x_{2 k+2}\right)\right] / 2\right\} } \\
= & k \max \left\{G_{2}\left(y_{2 k+1}, y_{2 k+1}, y_{2 k}\right), G_{2}\left(y_{2 k+2}, y_{2 k+2}, y_{2 k+1}\right),\right. \\
& \left.G_{2}\left(y_{2 k+1}, y_{2 k+1}, y_{2 k}\right),\left[G_{2}\left(y_{2 k+2}, y_{2 k+2}, y_{2 k}\right)+G_{2}\left(y_{2 k+1}, y_{2 k+1}, y_{2 k+1}\right)\right] / 2\right\} \\
\leq & k \max \left\{G_{2}\left(y_{2 k+1}, y_{2 k+1}, y_{2 k}\right), G_{2}\left(y_{2 k+2}, y_{2 k+2}, y_{2 k+1}\right),\right. \\
& {\left.\left[G_{2}\left(y_{2 k+2}, y_{2 k+2}, y_{2 k+1}\right)+G_{2}\left(y_{2 k+1}, y_{2 k+1}, y_{2 k}\right)\right] / 2\right\} } \\
\leq & k \max \left\{G_{1}\left(y_{2 k+1}, y_{2 k+1}, y_{2 k}\right), G_{1}\left(y_{2 k+2}, y_{2 k+2}, y_{2 k+1}\right)\right\} \\
= & k \max \left\{G_{1}\left(y_{n}, y_{n}, y_{n-1}\right), G_{1}\left(y_{n+1}, y_{n+1}, y_{n}\right)\right\},
\end{aligned}
$$

that is

$$
G_{1}\left(y_{n+1}, y_{n+1}, y_{n}\right) \leq k G_{1}\left(y_{n}, y_{n}, y_{n-1}\right)
$$

for all $n \in \mathbb{N}$. Continuing the above process, we have

$$
G_{1}\left(y_{n+1}, y_{n+1}, y_{n}\right) \leq k^{n} G_{1}\left(y_{1}, y_{1}, y_{0}\right)
$$

for all $n \in \mathbb{N}$. Thus, for all $n, m \in \mathbb{N}$ with $m>n$, we have

$$
\begin{aligned}
& G_{1}\left(y_{m}, y_{m}, y_{n}\right) \\
& \quad \leq G_{1}\left(y_{n}, y_{n+1}, y_{n+1}\right)+G_{1}\left(y_{n+1}, y_{n+2}, y_{n+2}\right)+\cdots+G_{1}\left(y_{m-1}, y_{m}, y_{m}\right) \\
& \leq k^{n} G_{1}\left(y_{0}, y_{1}, y_{1}\right)+k^{n+1} G_{1}\left(y_{0}, y_{1}, y_{1}\right)+\cdots+k^{m-1} G_{1}\left(y_{0}, y_{1}, y_{1}\right) \\
& \quad=k^{n} G_{1}\left(y_{0}, y_{1}, y_{1}\right) \sum_{i=0}^{m-n-1} k^{i} \\
& \quad \leq \frac{k^{n}}{1-k} G_{1}\left(y_{0}, y_{1}, y_{1}\right)
\end{aligned}
$$

and so $G_{1}\left(y_{n}, y_{m}, y_{m}\right) \rightarrow 0$ as $m, n \rightarrow \infty$. Hence $\left\{y_{n}\right\}$ is a $G$-Cauchy sequence in $X$. Since $X$ is $G_{1}$-complete, there exists a point $z \in X$ such that $\lim _{n \rightarrow \infty} y_{n}=z$. Consequently, we have

$$
\lim _{n \rightarrow \infty} y_{2 n+1}=\lim _{n \rightarrow \infty} f x_{2 n+1}=\lim _{n \rightarrow \infty} T x_{2 n+2}=z
$$

and

$$
\lim _{n \rightarrow \infty} y_{2 n}=\lim _{n \rightarrow \infty} g x_{2 n}=\lim _{n \rightarrow \infty} S x_{2 n+1}=z
$$

If $S$ is continuous and $\{f, S\}$ is compatible, then

$$
\begin{aligned}
& \lim _{n \rightarrow \infty} S^{2} x_{2 n+1}=S z, \\
& \lim _{n \rightarrow \infty} f S x_{2 n+1}=\lim _{n \rightarrow \infty} S f x_{2 n+1}=S z .
\end{aligned}
$$


Since $S x_{2 n+1}=g x_{2 n} \preceq x_{2 n},(2.1)$ gives

$$
\begin{aligned}
& G_{1}\left(f S x_{2 n+1}, f S x_{2 n+1}, g x_{2 n}\right) \\
& \leq k \max \left\{G_{2}\left(S S x_{2 n+1}, S S x_{2 n+1}, T x_{2 n}\right), G_{2}\left(f S x_{2 n+1}, f S x_{2 n+1}, S S x_{2 n+1}\right),\right. \\
& G_{2}\left(g x_{2 n}, g x_{2 n}, T x_{2 n}\right), \\
& {\left.\left[G_{2}\left(f S x_{2 n+2}, f S x_{2 n+2}, T x_{2 n}\right)+G_{2}\left(g x_{2 n}, g x_{2 n}, S S x_{2 n+1}\right)\right] / 2\right\} . }
\end{aligned}
$$

Taking the limit as $n \rightarrow \infty$, we obtain

$$
\begin{aligned}
G_{1}(S z, S z, z) \leq & k \max \left\{G_{2}(S z, S z, z), G_{2}(S z, S z, S z), G_{2}(z, z, z),\right. \\
& {\left.\left[G_{2}(S z, S z, z)+G_{2}(z, z, S z)\right] / 2\right\} } \\
\leq & k \max \left\{G_{1}(S z, S z, z),\left[G_{1}(S z, S z, z)+G_{1}(z, z, S z)\right] / 2\right\} \\
= & \frac{k}{2}\left[G_{1}(S z, S z, z)+G_{1}(z, z, S z)\right],
\end{aligned}
$$

which further implies that

$$
G_{1}(S z, S z, z) \leq h G_{1}(z, z, S z)
$$

where $h=\frac{k}{2-k}$. Obviously, $0 \leq h<1$.

Similarly, we obtain

$$
G_{1}(S z, z, z) \leq h G_{1}(z, S z, S z)
$$

From (2.5) and (2.6), we have

$$
G_{1}(S z, S z, z) \leq h^{2} G_{1}(z, S z, S z)
$$

and so $S z=z$ since $0 \leq h^{2}<1$. Since $g x_{2 n} \preceq x_{2 n}$ and $g x_{2 n} \rightarrow z$ as $n \rightarrow \infty$ implies $z \preceq x_{2 n}$, it follows from (2.1) that

$$
\begin{aligned}
& G_{1}\left(f z, f z, g x_{2 n}\right) \\
& \leq k \max \left\{G_{2}\left(S z, S z, T x_{2 n}\right), G_{2}(f z, f z, S z), G_{2}\left(g x_{2 n}, g x_{2 n}, T x_{2 n}\right),\right. \\
& {\left.\left[G_{2}\left(f z, f z, T x_{2 n}\right)+G_{2}\left(g x_{2 n}, g x_{2 n}, S z\right)\right] / 2\right\} } \\
&= k \max \left\{G_{2}\left(z, z, T x_{2 n}\right), G_{2}(f z, f z, z), G_{2}\left(g x_{2 n}, g x_{2 n}, T x_{2 n}\right),\right. \\
& {\left.\left[G_{2}\left(f z, f z, T x_{2 n}\right)+G_{2}\left(g x_{2 n}, g x_{2 n}, z\right)\right] / 2\right\}, }
\end{aligned}
$$

which, taking the limit as $n \rightarrow \infty$, gives

$$
\begin{aligned}
G_{1}(f z, f z, z) \leq & k \max \left\{G_{2}(z, z, z), G_{2}(f z, f z, z), G_{2}(z, z, z),\right. \\
& {\left.\left[G_{2}(f z, f z, z)+G_{2}(z, z, z)\right] / 2\right\} } \\
\leq & k G_{1}(f z, f z, z) .
\end{aligned}
$$


Similarly, we obtain

$$
G_{1}(f z, z, z) \leq k G_{1}(z, f z, f z)
$$

Therefore, by using the above two inequalities, we have $f z=z$.

Since $f(X) \subseteq T(X)$, there exists a point $v \in X$ such that $f z=T v$. Since $v \preceq T v=f z \preceq z$, it follows from (2.1) that

$$
\begin{aligned}
G_{1}(f z, f z, g v) \leq & k \max \left\{G_{2}(S z, S z, T v), G_{2}(f z, f z, S z), G_{2}(g v, g v, T v),\right. \\
& {\left.\left[G_{2}(f z, f z, T v)+G_{2}(g v, g v, S z)\right] / 2\right\} } \\
= & k \max \left\{G_{2}(f z, f z, f z), G_{2}(f z, f z, f z), G_{2}(g v, g v, f z),\right. \\
& {\left.\left[G_{2}(f z, f z, f z)+G_{2}(g v, g v, f z)\right] / 2\right\} } \\
\leq & k G_{1}(f z, g v, g v) .
\end{aligned}
$$

Similarly, we get

$$
G_{1}(f z, g v, g v) \leq k G_{1}(f z, f z, g v)
$$

Thus (2.9) and (2.10) imply $f z=g \nu$. Since $g$ and $T$ are weakly compatible, we have $g z=$ $g f z=g T v=T g v=T f z=T z$, and so $z$ is the coincidence point of $g$ and $T$.

Now, from (2.1), we have

$$
\begin{aligned}
G_{1}(z, z, g z)= & G_{1}(f z, f z, g z) \\
\leq & k \max \left\{G_{2}(S z, S z, T z), G_{2}(f z, f z, S z), G_{2}(g z, g z, T z),\right. \\
& {\left.\left[G_{2}(f z, f z, T z)+G_{2}(g z, g z, S z)\right] / 2\right\} } \\
= & k \max \left\{G_{2}(z, z, g z), G_{2}(z, z, z), G_{2}(g z, g z, g z),\right. \\
& {\left.\left[G_{2}(z, z, g z)+G_{2}(g z, g z, z)\right] / 2\right\} } \\
= & k \max \left\{G_{2}(z, z, g z),\left[G_{2}(z, z, g z)+G_{2}(g z, g z, z)\right] / 2\right\} \\
\leq & \frac{k}{2}\left[G_{1}(z, z, g z)+G_{1}(g z, g z, z)\right],
\end{aligned}
$$

that is,

$$
G_{1}(z, z, g z) \leq h G_{1}(g z, g z, z),
$$

where $h=\frac{k}{2-k}$. Obviously, $0 \leq h<1$. Using (2.2), we have

$$
G_{1}(z, g z, g z) \leq h G_{1}(z, z, g z) \text {. }
$$

Combining the above two inequalities, we get

$$
G_{1}(z, z, g z) \leq h^{2} G_{1}(z, z, g z)
$$


and so $z=g z$. Therefore, $f z=g z=S z=T z=z$. The proof is similar when $f$ is continuous. Similarly, if (b) holds, then the result follows.

Now, suppose that the set of common fixed points of $f, g, S$ and $T$ is well ordered. We show that a common fixed point of $f, g, S$ and $T$ is unique. Let $u$ be another common fixed point of $f, g, S$ and $T$. Then, from (2.1), we have

$$
\begin{aligned}
G_{1}(z, z, u)= & G_{1}(f z, f z, g u) \\
\leq & k \max \left\{G_{2}(S z, S z, T u), G_{2}(f z, f z, S z), G_{2}(g u, g u, T u),\right. \\
& {\left.\left[G_{2}(f z, f z, T u)+G_{2}(g u, g u, S z)\right] / 2\right\} } \\
= & k \max \left\{G_{2}(z, z, u), G_{2}(z, z, z), G_{2}(u, u, u),\right. \\
& {\left.\left[G_{2}(z, z, u)+G_{2}(u, u, z)\right] / 2\right\} } \\
= & \frac{k}{2}\left[G_{2}(z, z, u)+G_{2}(u, u, z)\right] \\
\leq & \frac{1}{2} G_{1}(z, z, u)+\frac{k}{2} G_{1}(u, u, z),
\end{aligned}
$$

that is,

$$
G_{1}(z, z, u) \leq k G_{1}(z, u, u)
$$

Similarly, using (2.2), we obtain

$$
G_{1}(z, u, u) \leq k G_{1}(z, z, u) .
$$

Combining the above two inequalities, we get

$$
G_{1}(z, z, u) \leq k^{2} G_{1}(z, z, u)
$$

and hence $z=u$.

The converse follows immediately. This completes the proof.

Example 2.2 Let $X=\{0,1,2,3\}$ be endowed with the usual ordering and $G_{1}, G_{2}$ be two $G$-metrics on $X$ defined by Table 2 . Then $G_{1}$ and $G_{2}$ are non-symmetric since $G_{1}(1,1,0) \neq$

\section{Table 2 Two G-metrices}

\begin{tabular}{ccc}
\hline $\boldsymbol{( x , y , z )}$ & $\mathbf{G}_{\mathbf{1}}(\boldsymbol{x}, \boldsymbol{y}, \boldsymbol{z})$ & $\mathbf{G}_{\mathbf{2}}(\boldsymbol{x}, \boldsymbol{y}, \boldsymbol{z})$ \\
\hline $\begin{array}{c}(0,0,0),(1,1,1),(2,2,2),(3,3,3), \\
(0,0,2),(0,2,0),(2,0,0),(0,2,2),(2,0,2),(2,2,0),\end{array}$ & 4 & 0 \\
$(0,0,1),(0,1,0),(1,0,0),(0,0,3),(0,3,0),(3,0,0)$, & & \\
$(0,1,1),(1,0,1),(1,1,0),(0,3,3),(3,0,3),(3,3,0)$, & 8 & 6 \\
$(1,1,2),(1,2,1),(2,1,1),(1,2,2),(2,1,2),(2,2,1)$, & & \\
$(1,1,3),(1,3,1),(3,1,1),(1,3,3),(3,1,3),(3,3,1)$, & & \\
$(2,2,3),(2,3,2),(3,2,2),(2,3,3),(3,2,3),(3,3,2)$, & & \\
$(0,1,2),(0,1,3),(0,2,1),(0,2,3),(0,3,1),(0,3,2)$, & 8 & \\
$(1,0,2),(1,0,3),(1,2,0),(1,2,3),(1,3,0),(1,3,2)$, & & \\
$(2,0,1),(2,0,3),(2,1,0),(2,1,3),(2,3,0),(2,3,1)$, & & \\
$(3,0,1),(3,0,2),(3,1,0),(3,1,2),(3,2,0),(3,2,1)$, & & \\
\hline
\end{tabular}


Table 3 Self maps

\begin{tabular}{ccccc}
\hline $\boldsymbol{x}$ & $\boldsymbol{f ( x )}$ & $\boldsymbol{g ( x )}$ & $\boldsymbol{S ( x )}$ & $\boldsymbol{T ( x )}$ \\
\hline 0 & 0 & 0 & 0 & 0 \\
1 & 0 & 0 & 2 & 2 \\
2 & 0 & 2 & 2 & 3 \\
3 & 0 & 0 & 3 & 3
\end{tabular}

Table 4 Dominated and dominating maps

\begin{tabular}{lllll}
\hline $\boldsymbol{x} \in \boldsymbol{X}$ & $\boldsymbol{f}$ is dominated & $\boldsymbol{g}$ is dominated & $\boldsymbol{S}$ is dominating & $\boldsymbol{T}$ is dominating \\
\hline$x=0$ & $f(0)=0$ & $g(0)=0$ & $0=S(0)$ & $0=T(0)$ \\
$x=1$ & $f(1)=0<1$ & $g(1)=0<1$ & $1<2=S(1)$ & $1<2=T(1)$ \\
$x=2$ & $f(2)=0<2$ & $g(2)=2$ & $2=S(2)$ & $2<3=T(2)$ \\
$x=3$ & $f(3)=0<3$ & $g(3)=0<3$ & $3=S(3)$ & $3=T(3)$ \\
\hline
\end{tabular}

$G_{1}(1,0,0)$ and $G_{2}(1,1,0) \neq G_{2}(1,0,0)$ with $G_{2}(x, y, z) \leq G_{1}(x, y, z)$ for all $x, y, z \in X$. Let $f, g, S, T: X \rightarrow X$ be the mappings defined by Table 3. Clearly, $f(X) \subseteq T(X), g(X) \subseteq S(X)$, $f, g$ are dominated mappings and $S, T$ are dominating mappings, see Table 4.

Now, we shall show that for all comparable $x, y \in X,(2.1)$ and (2.2) are satisfied with $k=\frac{3}{4} \in[0,1)$. Note that for all $x, y \in\{0,1,3\}, G(f x, f x, g y)=G(f x, g y, g y)=0$ and (2.1), (2.2) are satisfied obviously.

(1) When $x=0$ and $y=2$, then $f x=0, g y=2, S x=0, T y=3$ and so

$$
\begin{aligned}
G_{1}(f x, f x, g y)= & G_{1}(0,0,2)=4 \\
< & \frac{3}{4}(6)=\frac{3}{4} G_{2}(2,2,3)=\frac{3}{4} G_{2}(g y, g y, T y) \\
\leq & k \max \left\{G_{2}(S x, S x, T y), G_{2}(f x, f x, S x), G_{2}(g y, g y, T y),\right. \\
& {\left.\left[G_{2}(f x, f x, T y)+G_{2}(g y, g y, S x)\right] / 2\right\} }
\end{aligned}
$$

and

$$
\begin{aligned}
G_{1}(f x, g y, g y)= & G_{1}(0,2,2)=4 \\
< & \frac{3}{4}(6)=\frac{3}{4} G_{2}(2,3,3)=\frac{3}{4} G_{2}(g y, T y, T y) \\
\leq & k \max \left\{G_{2}(S x, T y, T y), G_{2}(f x, S x, S x), G_{2}(g y, T y, T y),\right. \\
& {\left.\left[G_{2}(f x, T y, T y)+G_{2}(g y, S x, S x)\right] / 2\right\} . }
\end{aligned}
$$

(2) When $x=1$ and $y=2$, then $f x=0, g y=2, S x=2, T y=3$ and so

$$
\begin{aligned}
G_{1}(f x, f x, g y)= & G_{1}(0,0,2)=4 \\
< & \frac{3}{4}(6)=\frac{3}{4} G_{2}(2,2,3)=\frac{3}{4} G_{2}(g y, g y, T y) \\
\leq & k \max \left\{G_{2}(S x, S x, T y), G_{2}(f x, f x, S x), G_{2}(g y, g y, T y),\right. \\
& {\left.\left[G_{2}(f x, f x, T y)+G_{2}(g y, g y, S x)\right] / 2\right\} }
\end{aligned}
$$


and

$$
\begin{aligned}
G_{1}(f x, g y, g y)= & G_{1}(0,2,2)=4 \\
< & \frac{3}{4}(6)=\frac{3}{4} G_{2}(2,3,3)=\frac{3}{4} G_{2}(g y, T y, T y) \\
\leq & k \max \left\{G_{2}(S x, T y, T y), G_{2}(f x, S x, S x), G_{2}(g y, T y, T y),\right. \\
& {\left.\left[G_{2}(f x, T y, T y)+G_{2}(g y, S x, S x)\right] / 2\right\} . }
\end{aligned}
$$

(3) When $x=2$ and $y=2$, then $f x=0, g y=2, S x=2, T y=3$ and so

$$
\begin{aligned}
G_{1}(f x, f x, g y)= & G_{1}(0,0,2)=4 \\
< & \frac{3}{4}(6)=\frac{3}{4} G_{2}(2,2,3)=\frac{3}{4} G_{2}(S x, S x, T y) \\
\leq & k \max \left\{G_{2}(S x, S x, T y), G_{2}(f x, f x, S x), G_{2}(g y, g y, T y),\right. \\
& {\left.\left[G_{2}(f x, f x, T y)+G_{2}(g y, g y, S x)\right] / 2\right\} }
\end{aligned}
$$

and

$$
\begin{aligned}
G_{1}(f x, g y, g y)= & G_{1}(0,2,2)=4 \\
< & \frac{3}{4}(6)=\frac{3}{4} G_{2}(2,3,3)=\frac{3}{4} G_{2}(S x, T y, T y) \\
\leq & k \max \left\{G_{2}(S x, T y, T y), G_{2}(f x, S x, S x), G_{2}(g y, T y, T y),\right. \\
& {\left.\left[G_{2}(f x, T y, T y)+G_{2}(g y, S x, S x)\right] / 2\right\} . }
\end{aligned}
$$

(4) Finally, when $x=3$ and $y=2$, then $f x=0, g y=2, S x=3, T y=3$ and so

$$
\begin{aligned}
G_{1}(f x, f x, g y)= & G_{1}(0,0,2)=4 \\
< & \frac{3}{4}(6)=\frac{3}{4} G_{2}(2,2,3)=\frac{3}{4} G_{2}(g y, g y, T y) \\
\leq & k \max \left\{G_{2}(S x, S x, T y), G_{2}(f x, f x, S x), G_{2}(g y, g y, T y),\right. \\
& {\left.\left[G_{2}(f x, f x, T y)+G_{2}(g y, g y, S x)\right] / 2\right\} }
\end{aligned}
$$

and

$$
\begin{aligned}
G_{1}(f x, g y, g y)= & G_{1}(0,2,2)=4 \\
< & \frac{3}{4}(6)=\frac{3}{4} G_{2}(2,3,3)=\frac{3}{4} G_{2}(g y, T y, T y) \\
\leq & k \max \left\{G_{2}(S x, T y, T y), G_{2}(f x, S x, S x), G_{2}(g y, T y, T y),\right. \\
& {\left.\left[G_{2}(f x, T y, T y)+G_{2}(g y, S x, S x)\right] / 2\right\} . }
\end{aligned}
$$

Thus, for all cases, the contractions (2.1) and (2.2) are satisfied. Hence all of the conditions of Theorem 2.1 are satisfied. Moreover, 0 is the unique common fixed point of $f, g, S$ and $g$. 
If we consider the same set equipped with two metrics given by $d_{1}(x, y)=|x-y|$ and $d_{2}(x, y)=\frac{1}{2}|x-y|$ for all $x, y \in X$, then for $x=1$ and $y=2$, we have

$$
\begin{aligned}
d_{1}(f x, g y) & =d_{1}(0,2)=2 \not \leq 2 k \\
& \leq k \max \left\{d_{2}(2,3), d_{2}(0,2), d_{2}(2,3),\left[d_{2}(0,3)+d_{2}(2,2)\right] / 2\right\} \\
& =k \max \left\{d_{2}(S x, T y), d_{2}(f x, S x), d_{2}(g y, T y),\left[d_{2}(f x, T y)+d_{2}(g y, S x)\right] / 2\right\}
\end{aligned}
$$

for any $k \in[0,1)$. So corresponding results in ordinary metric spaces cannot be applied in this case.

Theorem 2.1 can be viewed as an extension of Theorem 2.1 of [8] to the case of two ordered G-metric spaces.

Since the class of weakly compatible mappings includes $R$-weakly commuting mappings, Theorem 2.1 generalizes the comparable results in [8].

Corollary 2.3 Let $(X, \preceq)$ be a partially ordered set and $G_{1}, G_{2}$ be two $G$-metrics on $X$ such that $G_{2}(x, y, z) \leq G_{1}(x, y, z)$ for all $x, y, z \in X$ with a complete metric $G_{1}$ on $X$. Suppose that $f, g, S$ and $T$ are self-mappings on $X$ satisfying the following properties:

$$
\begin{aligned}
G_{1}(f x, f x, g y) \leq & a_{1} G_{2}(S x, S x, T y)+a_{2} G_{2}(S x, S x, f x)+a_{3} G_{2}(T y, T y, g y) \\
& +a_{4}\left[G_{2}(S x, S x, g y)+G_{2}(T y, T y, f x)\right]
\end{aligned}
$$

and

$$
\begin{aligned}
G_{1}(f x, g y, g y) \leq & a_{1} G_{2}(S x, T y, T y)+a_{2} G_{2}(S x, f x, f x)+a_{3} G_{2}(T y, g y, g y) \\
& +a_{4}\left[G_{2}(S x, g y, g y)+G_{2}(T y, f x, f x)\right]
\end{aligned}
$$

for all comparable $x, y \in X$, where $a_{1}+a_{2}+a_{3}+2 a_{4}<1$. Suppose that $f(X) \subseteq T(X)$, $g(X) \subseteq S(X)$ and $f, g$ are dominated mappings and $S$, $T$ are dominating mappings. If, for any nonincreasing sequence $\left\{x_{n}\right\}$ with $y_{n} \preceq x_{n}$ for all $n \in \mathbb{N}, y_{n} \rightarrow u$ implies that $u \preceq x_{n}$ and either

(a) $f, S$ are compatible, $f$ or $S$ is continuous and $g, T$ are weakly compatible or

(b) $g$, $T$ are compatible, $g$ or $T$ is continuous and $f$, $S$ are weakly compatible, then $f, g$, S and T have a common fixed point in X. Moreover, the set of common fixed points of $f, g, S$ and $T$ is well-ordered if and only if $f, g, S$ and $T$ have one and only one common fixed point in $X$.

Example 2.4 Let $X=[0,1]$ be endowed with the usual ordering and $G_{1}, G_{2}$ be two $G$ metrics on $X$ given in [13]:

$$
\begin{aligned}
& G_{1}(a, b, c)=|a-b|+|b-c|+|c-a|, \\
& G_{2}(a, b, c)=\frac{1}{2}[|a-b|+|b-c|+|c-a|] .
\end{aligned}
$$


Define the mappings $f, g, S, T: X \rightarrow X$ as

$$
f x=\frac{x}{12}, \quad g x= \begin{cases}\frac{x}{4} & \text { if } x \in\left[0, \frac{1}{2}\right), \quad S(x)=\frac{3 x}{2}, \quad T(x)=\frac{5 x}{2} \\ \frac{x}{6} & \text { if } x \in\left[\frac{1}{2}, 1\right),\end{cases}
$$

for all $x \in X$. Clearly, $f, g$ are dominated mappings and $S, T$ are dominating mappings with $f(X) \subseteq T(X)$ and $g(X) \subseteq S(X)$. Also, $f, S$ are compatible, $f$ is continuous and $g, T$ are weakly compatible. Now, for all comparable $x, y \in X$, we check the following cases:

(1) If $x, y \in\left[0, \frac{1}{2}\right)$, then we have

$$
\begin{aligned}
G_{1}(f x, f x, g y)= & \frac{1}{12}|x-3 y| \leq \frac{1}{12}(x+3 y) \\
\leq & \frac{3}{10}\left(\frac{17}{12} x\right)+\frac{3}{10}\left(\frac{9}{4} y\right) \\
= & a_{2} G_{2}(f x, f x, S x)+a_{3} G_{2}(g y, g y, T y) \\
\leq & a_{1} G_{2}(S x, S x, T y)+a_{2} G_{2}(f x, f x, S x)+a_{3} G_{2}(g y, g y, T y) \\
& +a_{4}\left[G_{2}(f x, f x, T y)+G_{2}(g y, g y, S x)\right] .
\end{aligned}
$$

(2) If $x \in\left[0, \frac{1}{2}\right)$ and $y \in\left[\frac{1}{2}, 1\right]$, then we have

$$
\begin{aligned}
G_{1}(f x, f x, g y)= & \frac{1}{12}|x-2 y| \leq \frac{1}{12}(x+2 y) \\
\leq & \frac{3}{10}\left(\frac{17}{12} x\right)+\frac{3}{10}\left(\frac{14}{6} y\right) \\
= & a_{2} G_{2}(f x, f x, S x)+a_{3} G_{2}(g y, g y, T y) \\
\leq & a_{1} G_{2}(S x, S x, T y)+a_{2} G_{2}(f x, f x, S x)+a_{3} G_{2}(g y, g y, T y) \\
& +a_{4}\left[G_{2}(f x, f x, T y)+G_{2}(g y, g y, S x)\right] .
\end{aligned}
$$

(3) If $y \in\left[0, \frac{1}{2}\right)$ and $x \in\left[\frac{1}{2}, 1\right]$, then we have

$$
\begin{aligned}
G_{1}(f x, f x, g y)= & \frac{1}{12}|x-3 y| \leq \frac{1}{12}(x+3 y) \\
\leq & \frac{3}{10}\left(\frac{17}{12} x\right)+\frac{3}{10}\left(\frac{9}{4} y\right) \\
= & a_{2} G_{2}(f x, f x, S x)+a_{3} G_{2}(g y, g y, T y) \\
\leq & a_{1} G_{2}(S x, S x, T y)+a_{2} G_{2}(f x, f x, S x)+a_{3} G_{2}(g y, g y, T y) \\
& +a_{4}\left[G_{2}(f x, f x, T y)+G_{2}(g y, g y, S x)\right] .
\end{aligned}
$$

(4) If $x, y \in\left[\frac{1}{2}, 1\right]$, then we obtain

$$
\begin{aligned}
G_{1}(f x, f x, g y) & =\frac{1}{12}|x-2 y| \leq \frac{1}{12}(x+2 y) \\
& \leq \frac{3}{10}\left(\frac{17}{12} x\right)+\frac{3}{10}\left(\frac{14}{6} y\right)
\end{aligned}
$$




$$
\begin{aligned}
= & a_{2} G_{2}(f x, f x, S x)+a_{3} G_{2}(g y, g y, T y) \\
\leq & a_{1} G_{2}(S x, S x, T y)+a_{2} G_{2}(f x, f x, S x)+a_{3} G_{2}(g y, g y, T y) \\
& +a_{4}\left[G_{2}(f x, f x, T y)+G_{2}(g y, g y, S x)\right] .
\end{aligned}
$$

Thus (2.13) is satisfied with $a_{1}=a_{4}=\frac{1}{10}$ and $a_{2}=a_{3}=\frac{3}{10}$, where $a_{1}+a_{2}+a_{3}+2 a_{4}<1$. Similarly, (2.14) is satisfied. Thus all the conditions of Corollary 2.3 are satisfied. Moreover, 0 is the unique common fixed point of $f$ and $g$.

\section{Application}

Let $X=L^{2}(\Omega)$, the set of comparable functions on $\Omega$ whose square is integrable on $\Omega$ where $\Omega=[0,1]$, be a bounded set in $\mathbb{R}$. We endow $X$ with the partial ordered $\preceq$ given by: $x, y \in X, x \preceq y \Leftrightarrow x(t) \leq y(t)$, for all $t \in \Omega$. We consider the integral equations

$$
\begin{aligned}
& x(t)=\int_{\Omega} q_{1}(t, s, x(s)) d s-c(t), \\
& y(t)=\int_{\Omega} q_{2}(t, s, y(s)) d s-c(t),
\end{aligned}
$$

where $q_{1}, q_{2}: \Omega \times \Omega \times \mathbb{R} \rightarrow \mathbb{R}$ and $c: \Omega \rightarrow \mathbb{R}^{+}$, to be given continuous mappings. Recently, Abbas et al. [35] obtained a common solution of integral equations (3.1) as an application of their results in the setup of ordered generalized metric spaces. Here we study a sufficient condition for the existence of a common solution of integral equations in the framework of two generalized metric spaces. Define $G_{1}, G_{2}: X \times X \times X \rightarrow \mathbb{R}^{+}$by

$$
\begin{aligned}
& G_{1}(x, y, z)=\sup _{t \in \Omega}|x(t)-y(t)|+\sup _{t \in \Omega}|y(t)-z(t)|+\sup _{t \in \Omega}|z(t)-x(t)|, \\
& G_{2}(x, y, z)=\frac{1}{2}\left[\sup _{t \in \Omega}|x(t)-y(t)|+\sup _{t \in \Omega}|y(t)-z(t)|+\sup _{t \in \Omega}|z(t)-x(t)|\right] .
\end{aligned}
$$

Obviously, $G_{2}(x, y, z) \leq G_{1}(x, y, z)$ for all $x, y, z \in X$. Suppose that the following hypotheses hold:

(i) For each $s, t \in \Omega$,

$$
\int_{\Omega} q_{1}(t, s, u(s)) d s \leq u(s)
$$

and

$$
\int_{\Omega} q_{2}(t, s, u(s)) d s \leq u(s)
$$

hold.

(ii) There exists $r: \Omega \rightarrow \Omega$ such that

$$
\int_{\Omega}\left|q_{1}(t, s, u(t))-q_{2}(t, s, v(t))\right| d t \leq r(t)|u(t)-v(t)|
$$

for each $s, t \in \Omega$ with $\sup _{t \in \Omega} r(t) \leq k$ where $k \in[0,1)$.

Then the integral equations (3.1) have a common solution in $L^{2}(\Omega)$. 
Proof Define $f x(t)=\int_{\Omega} q_{1}(t, s, x(t)) d t-c(t)$ and $g x(t)=\int_{\Omega} q_{2}(t, s, x(t)) d t-c(t)$. As $f x(t) \leq$ $x(t)$ and $g x(t) \leq x(t)$, so $f$ and $g$ are dominated maps. Now, for all comparable $x, y \in X$,

$$
\begin{aligned}
G_{1}(f x, f x, g y)= & 2 \sup _{t \in \Omega}|f x(t)-g y(t)| \\
= & 2 \sup _{t \in \Omega}\left|\int_{\Omega} q_{1}(t, s, x(t)) d t-\int_{\Omega} q_{2}(t, s, y(t)) d t\right| \\
\leq & 2 \sup _{t \in \Omega} \int_{\Omega}\left|q_{1}(t, s, x(t))-q_{2}(t, s, y(t))\right| d t \\
\leq & 2 \sup _{t \in \Omega} r(t)|x(t)-y(t)| \\
\leq & 2 k \sup _{t \in \Omega}|x(t)-y(t)| \\
= & k G_{2}(x, y, y) \\
\leq & k \max \left\{G_{2}(x, x, y), G_{2}(f x, f x, x), G_{2}(g y, g y, y),\right. \\
& {\left.\left[G_{2}(f x, f x, y)+G_{2}(g y, g y, x)\right] / 2\right\} . }
\end{aligned}
$$

Similarly,

$$
\begin{gathered}
G_{1}(f x, g y, g y) \leq k \max \left\{G_{2}(x, y, y), G_{2}(f x, x, x), G_{2}(g y, y, y),\right. \\
\left.\left[G_{2}(f x, y, y)+G_{2}(g y, x, x)\right] / 2\right\}
\end{gathered}
$$

is satisfied. Now we can apply Theorem 2.1 by taking $S$ and $T$ as identity maps to obtain the common solutions of integral equations (3.1) in $L^{2}(\Omega)$.

\section{Remarks}

(1) If we take $f=g$ in Theorem 2.1, then it generalizes Corollary 2.3 in [8] to a more general class of commuting mappings in the setup of two ordered G-metric spaces.

(2) If we take $S=T$ in Theorem 2.1, then Corollary 2.4 in [8] is a special case of Theorem 2.1.

(3) If $S=T=I_{X}$ (: the identity mapping on $X$ ) in Theorem 2.1, then we obtain Corollary 2.5 in [8] in a more general setup.

(4) Corollary 2.6 of [8] becomes a special case of Theorem 2.1 if we take $f=g$ and $S=$ $T=I_{X}$.

(5) A $G$-metric naturally induces a metric $d_{G}$ given by $d_{G}(x, y)=G(x, y, y)+G(x, x, y)$. If the G-metric is not symmetric, then the inequalities (2.1), (2.2), (2.13) and (2.14) do not reduce to any metric inequality with the metric $d_{G}$. Hence our results do not reduce to fixed point problems in the corresponding metric space $\left(X, \preceq, d_{G}\right)$.

Competing interests

The authors declare that they have no competing interests.

Authors' contributions

All authors read and approved the final manuscript.

\section{Acknowledgements}

The authors thank the referees for their appreciation and suggestions regarding this work. 


\section{References}

1. Mustafa, Z, Sims, B: A new approach to generalized metric spaces. J. Nonlinear Convex Anal. 7, 289-297 (2006)

2. Mustafa, Z, Obiedat, H, Awawdeh, F: Some fixed point theorem for mapping on complete G-metric spaces. Fixed Point Theory Appl. 2008, Article ID 189870 (2008)

3. Mustafa, Z, Sims, B: Fixed point theorems for contractive mappings in complete G-metric spaces. Fixed Point Theory Appl. 2009, Article ID 917175 (2009)

4. Mustafa, Z, Shatanawi, W, Bataineh, M: Existence of Fixed point Results in G-metric spaces. Int. J. Math. Math. Sci. 2009, Article ID 283028 (2009)

5. Mustafa, Z, Awawdeh, F, Shatanawi, W: Fixed point theorem for expansive mappings in G-metric spaces. Internat. J. Contemp. Math. Sci. 5, 2463-2472 (2010)

6. Abbas, M, Rhoades, BE: Common fixed point results for non-commuting mappings without continuity in generalized metric spaces. Appl. Math. Comput. 215, 262-269 (2009)

7. Abbas, M, Nazir, T, Radenović, S: Some periodic point results in generalized metric spaces. Appl. Math. Comput. 217, 4094-4099 (2010)

8. Abbas, M, Khan, SH, Nazir, T: Common fixed points of R-weakly commuting maps in generalized metric spaces. Fixed Point Theory Appl. 2011, 41 (2011)

9. Abbas, M, Cho, YJ, Nazir, T: Common fixed point theorems for four mappings in TVS-valued cone metric spaces. J. Math. Inequal. 5, 287-299 (2011)

10. Chugh, R, Kadian, T, Rani, A, Rhoades, BE: Property $p$ in G-metric spaces. Fixed Point Theory Appl. 2010, Article ID $401684(2010)$

11. Saadati, R, Vaezpour, SM, Vetro, P, Rhoades, BE: Fixed point theorems in generalized partially ordered G-metric spaces. Math. Comput. Model. 52, 797-801 (2010)

12. Shatanawi, W: Fixed point theory for contractive mappings satisfying $\Phi$-maps in G-metric spaces. Fixed Point Theory Appl. 2010, Article ID 181650 (2010)

13. Abbas, M, Khan, AR, Nazir, T: Coupled common fixed point results in two generalized metric spaces. Appl. Math. Comput. 217, 6328-6336 (2011)

14. Aydi, H, Damjanović, B, Samet, B, Shatanawi, W: Coupled fixed point theorems for nonlinear contractions in partially ordered G-metric spaces. Math. Comput. Model. 54, 2443-2450 (2011)

15. Chang, SS, Cho, YJ, Huang, NJ: Coupled fixed point theorems with applications. J. Korean Math. Soc. 33, 575-585 (1996)

16. Cho, YJ, He, G, Huang, NJ: The existence results of coupled quasi-solutions for a class of operator equations. Bull. Korean Math. Soc. 47, 455-465 (2010)

17. Cho, YJ, Rhoades, BE, Saadati, R, Samet, B, Shatanawi, W: Nonlinear coupled fixed point theorems in ordered generalized metric spaces with integral type. Fixed Point Theory Appl. 2012, 8 (2012). doi:10.1186/1687-1812-2012-8

18. Cho, YJ, Shah, MH, Hussain, N: Coupled fixed points of weakly F-contractive mappings in topological spaces. Appl. Math. Lett. 24, 1185-1190 (2011)

19. Choudhury, BS, Maity, P: Coupled fixed point results in generalized metric spaces. Math. Comput. Model. 54, 73-79 (2011)

20. Gordji, ME, Cho, YJ, Baghani, H: Coupled fixed point theorems for contractions in intuitionistic fuzzy normed spaces. Math. Comput. Model. 54, 1897-1906 (2011)

21. Sintunavarat, W, Cho, YJ, Kumam, P: Coupled coincidence point theorems for contractions without commutative condition in intuitionistic fuzzy normed spaces. Fixed Point Theory Appl. 2011, 81 (2011)

22. Ran, ACM, Reurings, MCB: A fixed point theorem in partially ordered sets and some application to matrix equations. Proc. Am. Math. Soc. 132, 1435-1443 (2004)

23. Nieto, JJ, Lopez, RR: Contractive mapping theorems in partially ordered sets and applications to ordinary differential equations. Order 22, 223-239 (2005)

24. Abbas, M, Nazir, T, Radenović, S: Common fixed points of four maps in partially ordered metric spaces. Appl. Math. Lett. 24, 1520-1526 (2011)

25. Cho, YJ, Saadati, R, Wang, S: Common fixed point theorems on generalized distance in order cone metric spaces. Comput. Math. Appl. 61, 1254-1260 (2011)

26. Guo, D, Cho, YJ, Zhu, J: Partial Ordering Methods in Nonlinear Problems. Nova Science Publishers, New York (2004)

27. Huang, NJ, Fang, YP, Cho, YJ: Fixed point and coupled fixed point theorems for multi-valued increasing operators in ordered metric spaces. In: Cho, YJ, Kim, JK, Kang, SM (eds.) Fixed Point Theory and Applications, vol. 3, pp. 91-98. Nova Science Publishers, New York (2002)

28. Nieto, JJ, Pouso, RL, Rodríguez-López, R: Fixed point theorems in ordered abstract sets. Proc. Am. Math. Soc. 135 2505-2517 (2007)

29. Nieto, JJ, Rodríguez-López, R: Existence and uniqueness of fixed points in partially ordered sets and applications to ordinary differential equations. Acta Math. Sin. Engl. Ser. 23, 2205-2212 (2007)

30. Sintunavarat, W, Cho, YJ, Kumam, P: Common fixed point theorems for c-distance in ordered cone metric spaces. Comput. Math. Appl. 62, 1969-1978 (2011)

31. Jungck, G: Compatible mappings and common fixed points. Int. J. Math. Math. Sci. 9, 771-779 (1986)

32. Kannan, R: Some results on fixed points. Bull. Calcutta Math. Soc. 60, $71-76$ (1968)

33. Ćirić, Lj: Generalized contractions and fixed-point theorems. Publ. Inst. Math. 12(26), 19-26 (1971)

34. Cirić, Lj: Fixed points for generalized multi-valued contractions. Mat. Vesnik 9(24), 265-272 (1972)

35. Abbas, M, Nazir, T, Radenović, S: Common fixed point of generalized weakly contractive maps in partially ordered G-metric spaces. Appl. Math. Comput. 218, 9383-9395 (2012

doi:10.1186/1687-1812-2012-139

Cite this article as: Abbas et al.: Common fixed points of Ćirić-type contractive mappings in two ordered generalized metric spaces. Fixed Point Theory and Applications 2012 2012:139. 